\title{
ELECTROCHEMICAL MICROSENSORS FOR DNA MUTATION DETECTION
}

\author{
Xing Yang, Chunnian Shi, R. Erik Holmlin, Shana O. Kelley, Michael G. Hill \\ GeneOhm Sciences, Inc. \\ 6146 Nancy Ridge Drive, Suite 101 \\ San Diego, CA 92121 \\ Donald M. Crothers \\ Department of Chemistry \\ Yale University \\ 225 Prospect Street \\ New Haven, CT 06511 \\ Jacqueline K. Barton \\ Department of Chemistry \\ Mail Code 127-77 \\ California Institute of Technology \\ Pasadena, CA 91125
}

\section{ABSTRACT}

We have developed a new electrochemical microsensor for DNA mutation detection. It is based on the unique properties of DNA-mediated electron transfer. Gold microelectrode arrays have been designed and fabricated on silicon substrates. Densely packed double-stranded DNA thin films on gold surfaces have been prepared and characterized. It has been shown that the redox-active intercalators bound to the DNA films exhibit well-resolved electrochemical responses. More importantly, it has also been demonstrated that DNA-mediated reduction of the bound intercalator depends critically on stacking within the double helical array. Based on difference in charge transport through doublestranded DNA rather than hybridization energy, we have demonstrated detection of point mutations in DNA duplexes irrespective of the DNA sequence composition, mutation position in the DNA duplexes, or mutation identity.

\section{INTRODUCTION}

The success of the human genome project (HGP) provides a starting point for understanding basic human genetic makeup. It is agreed that genetic variations, with SNP (single nucleotide polymorphisms, single-base variations in genetic codes) being the most common one, are not only responsible for human diversity but also carry significant medical differences in disease susceptibility and drug response. Reliable detection of such genetic mutations is critical for the study, diagnosis, and treatment of many human diseases.

To date, most assays proposed for large-scale genetic analysis rely on molecular recognition events associated with DNA hybridization $[\mathbf{1 , 2}]$ to catalogue sequence information. Applied to mutation detection, hybridization assays are inherently limited in specificity, especially in the case of SNPs when only one base in the DNA strand is mutated. Detection of a SNP in the test sequence requires a distinguishable difference in pairing energies between the probe sequence and a completely complementary versus mutated target strand. With only a single mutation in an extended oligonucleotide, these differences can be very small. Moreover, duplex stabilities for oligonucleotides of a fixed length can vary considerably as a function of base content, with GC-rich sequences significantly more stable than AT-rich analogues. As a consequence, detection of point mutations with libraries of immobilized oligonucleotides (where duplex-binding energies for adjacent probe sequences may vary significantly more than the differential binding energies of a particular probe with its complementary versus mutated test sequences) requires extensive manipulation of hybridization conditions as well as sophisticated deconvolution algorithms.
Monitoring charge transport through double-stranded DNA offers an alternative approach to the detection of point mutations. Photoinduced electron transfer through donor/acceptor-labeled duplexes has been observed in a variety of systems [3]. Significantly, DNA-mediated reactions show a weak dependence on distance but are exceptionally sensitive to the perturbations in the base stack: intervening bulges inhibit long-range photochemical guanine oxidation, and single-base mismatches markedly reduce photoinduced electron-transfer yields [3]. Thus, while single-base mismatches may cause only subtle changes in duplex stability and structure, they appear to induce significant perturbations in the electronic structure of the base-pair stack. This sensitivity of electron transfer to perturbation in the DNA duplex provides a complementary signaling mechanism for mutation detection.

Based on DNA-mediated electron transfer, here we present electrochemical microsensors for accurate, rapid, and low-cost detection of genetic mutations.

\section{DNA-MEDIATED ELECTRON TRANSFER}

Electrochemistry has been used extensively to investigate the kinetics of electron transfer through self-assembled monolayers on solid surfaces. Systems that feature redox-active head groups held at various distances by aliphatic alkanethiols or conjugated linkers have yielded important information regarding the ability of different media to promote long-range electronic coupling. To investigate DNA-mediated electron transfer, we have applied these methods to study redox-active intercalators bound at discrete sites within the individual helices of a DNA monolayer on gold.

We have previously developed techniques for assembling DNA duplexes derivatized at the 5 ' end with thiol-terminated linkers onto gold surfaces [4]. Electrochemical assays, radio active tagging experiments, and atomic force microscopy (AFM) all indicated that the duplexes form densely packed monlayers oriented in an upright position with respect to the gold surface [4] as shown in Figure 1. An average of surface coverage of 50

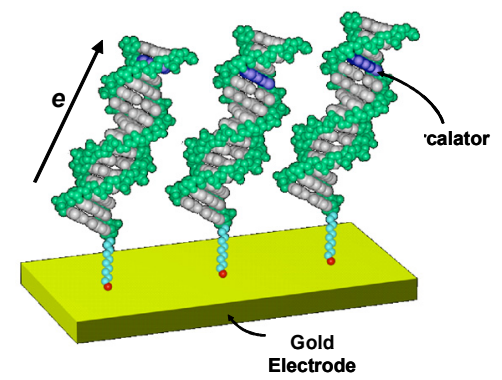

Figure 1. DNA-Modified Gold Electrode. 


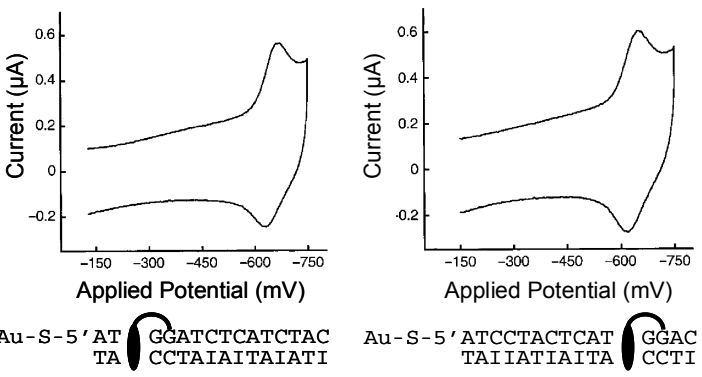

(a)

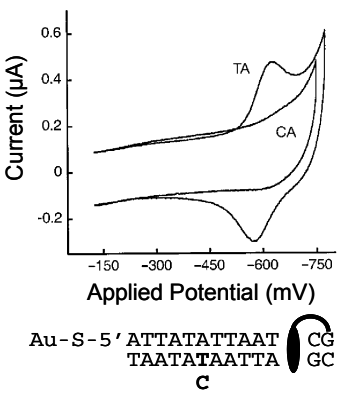

(b)

Figure 2. Voltammograms of Gold Electrodes Modified with DM-Cross-Linked Duplexes.

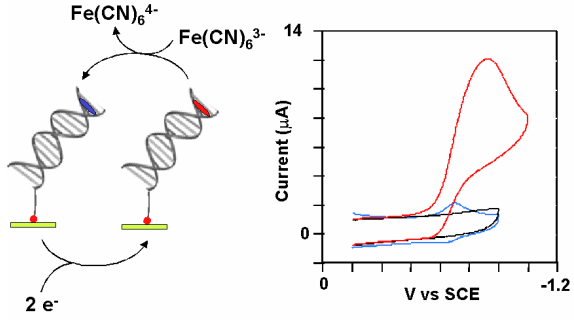

Figure 4. Electrocatalysis. $\mathrm{pmol} / \mathrm{cm}^{2}$ has been reported. Our work showed several unique features of double-stranded DNA films. Densely packed thiolmodified duplexes readily adsorb to gold with a high and apparently uniform surface coverage. Moreover, as long as electrochemical experiments are conducted at sufficiently negative potentials, the DNA duplexes are oriented in an upright position with respect to the metal surface. This arrangement helps direct charge transport through the double helix.

DNA modified electrodes exhibit very high affinities for DNA-binding substrates, and promote efficient electron transfer between the electrode and redox-active intercalators. To investigate charge transfer through DNA as a function of distance, we have site-specifically cross-linked a redox-active intercalator, daunomycin (DM), into the DNA films. DM undergoes a reversible reduction within the potential window of the monolayers. The site of intercalation was controlled in the duplexes by incorporating a single GC base step in otherwise A-T or inosine(I)-C sequences; as daunomycin requires the $\mathrm{N}-2$ of guanine for covalent cross-linking, the intercalator is constrained to these positions. Moving the GC-step along the duplex therefore provided a systematic variation in the location of the DM-binding site relative to the thiol-terminated linker. Cross-linking DM to the DNA in solution, and then depositing the labeled duplexes onto gold afforded a series of films in which the intercalator was linked quantitatively at a known separation from the electrode surface.

In the first experiment, films with 15-base-pair DM-labeled DNA duplexes were formed on gold electrodes $\left(0.02 \mathrm{~cm}^{2}\right.$, MF2014 from Bioanalytical Systems, Inc.) As shown in Figure 2 (a), with the controlled position of the GC base pair in the duplexes, the distance of DM from the gold surface was significantly different between the two duplexes ( $15 \AA$ versus $45 \AA$ ). However, efficient reduction of DM was observed regardless of its position along the 15 base-pair sequence. Not only were the intensities of the DM signals the same for each of the duplexes studied, but the

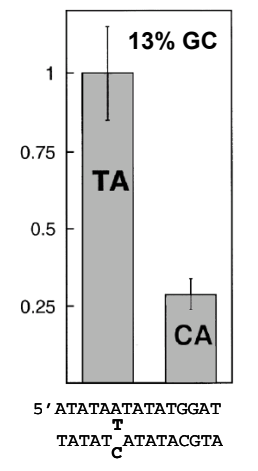

$T_{m}: 42$ vs. $40^{\circ} \mathrm{C}$
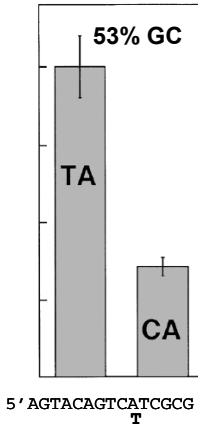
TCATGTCAG AGCGC

$\boldsymbol{T}_{\boldsymbol{m}}: \mathbf{5 8}$ vs. $5^{\circ} \mathrm{C}$

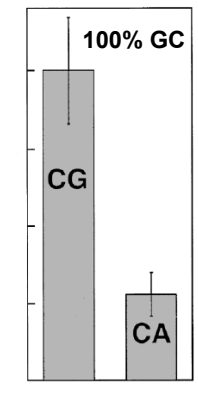

5' GGCGCCCGGCGCCGG ${ }_{\text {CCGC }}^{\mathbf{G}}$ GGCCGCGGCC

$\boldsymbol{T}_{\boldsymbol{m}}: 78$ vs. $74^{\circ} \mathrm{C}$
Figure 3. Sequence Independence. characteristic splitting between the catholic and anodic waves as a function of scan rate were essentially invariant throughout the entire series. Clearly within the time resolution of this experiment, increasing the through-helix DM/gold separation did not substantially affect the rate of electron transfer.

In the second experiment, a single site within the 15-base-pair duplex was mutated to produce a CA mismatch between the intercalated DM and the gold electrode surface. Although CA mismatches are known to cause only local disruptions in the DNA base stack with the base remaining intrahelical, the presence of this one-base change switched off the electrochemical response entirely, as shown in Figure 2 (b). In the related control experiment, sequences in which the positions of DM and CA mismatch were reversed (such that the mismatch was located above the DM relative to the gold) showed no diminution in the electrochemical response. Moreover, AFM images of the CAmutated sequences were indistinguishable from those of the fully base-paired analogues, revealing that the bulk structure of the DNA films was not significantly altered by the presence of the mismatch.

It is determined in the next experiment that the characteristic drop in coulometric signals for DM bound to DNA films containing CA mismatch compared to fully paired films is essentially invariant across AT-rich to GC-rich sequences tested under identical conditions. As shown in Figure 3, DNA duplexes with varied percentage of GC content were either fully base-paired or contain a single CA mismatch. Regardless of the sequence composition, and therefore over a wild range of duplex stabilities $\left(\Delta \mathrm{T}_{\mathrm{m}}=40^{\circ} \mathrm{C}\right)$, the $\mathrm{CA}$ mismatches contained with these duplexes were accurately detected.

Finally, although duplexes containing mismatches can be distinguished by direct voltammetry of redox-active intercalators, the absolute electrochemical signals are limited by the surface concentration of the intercalators $\left(\sim 50 \mathrm{pmol} / \mathrm{cm}^{2}\right)$. In order to increase the inherent sensitivity of this assay, we coupled the direct electron transfer to an electrocatalytic process involving a species freely diffusing in solution (Figure 4 (a)). Methylene blue (MB) was chosen as the intercalated catalyst with potassium ferricyanide as the solution substrate. Shown in Figure 4 (b), addition of micromolar $\mathrm{MB}$ to a $2.0 \mathrm{mM}$ ferricyanide solution caused a pronounced electrochemical signal at the DNA-modified electrode. Notably this signal comes at the reduction potential of MB and is completely irreversible: electrons flow from the Au electrode to the intercalated $\mathrm{MB}$ and then are accepted by ferricyanide in solution (thus no electrochemical oxidation peak is observed). Chemical oxidized MB is again available for electrochemical reduction and the catalytic cycle continues as long as the potential of the gold electrode is sufficiently negative to MB. This electrocatalysis process effectively amplifies the intercalator signal and improves the discrimination between signals obtained for mismatched versus base-paired duplex films. 


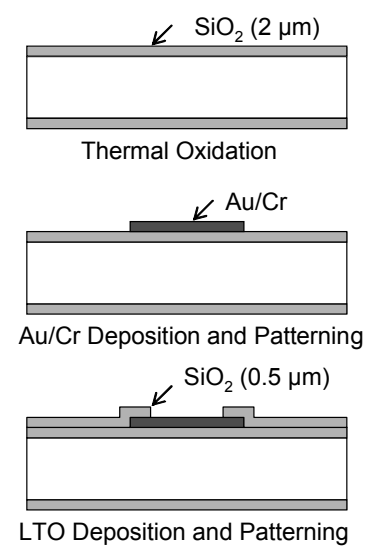

(a)

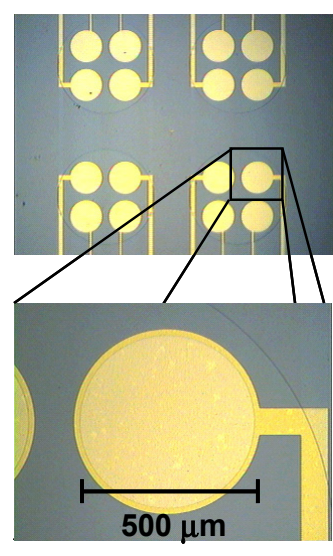

(b)
Figure 5. Sensor Fabrication Process.

Taken together, these experiments have established the basic understanding of DNA-mediated electron transfer and provided the foundation for developing chip-based electrochemical microsensors for DNA mutation detection.

\section{SENSOR DESIGN AND FABRICATION}

Fabricated on a silicon substrate, the sensor consists of an array of gold microelectrodes sandwiched between two layers of silicon dioxide. Microelectrodes with varied dimensions ranging from $0.1 \mathrm{~mm}$ to $1 \mathrm{~mm}$ in diameter and $500 \AA$ to $6500 \AA$ in thickness were designed and fabricated.

Shown in Figure 5 (a), the fabrication process started with 4 inch $<100>$ silicon wafers. First, a layer of $2 \mu \mathrm{m}$ silicon dioxide was thermally grown on the wafer at $1100{ }^{\circ} \mathrm{C}$. Then, a thin layer of $100 \AA$ chromium followed by a layer of gold were deposited by thermal evaporation. The thin chromium layer improved the adhesion of gold to the silicon oxide surface. In order to investigate its effect on sensor performance, the thickness of the gold layer was varied from $100 \AA$ to $6500 \AA$. Finally, another layer of $5000 \AA$ LPCVD silicon dioxide was deposited at $450{ }^{\circ} \mathrm{C}$ and patterned to expose the Au electrodes.

Figure 5 (b) shows an array of fabricated gold microelectrodes.

\section{EXPERIMENTS AND RESULTS}

\section{Gold Surface Preparation and Characterization}

Electrochemical cleaning - cyclic voltammetry of oxidation and reduction of the gold electrode in sulfuric acid - was used to remove any contaminant adsorbed physically or chemically on the gold surface and also qualitatively characterize the gold surface.

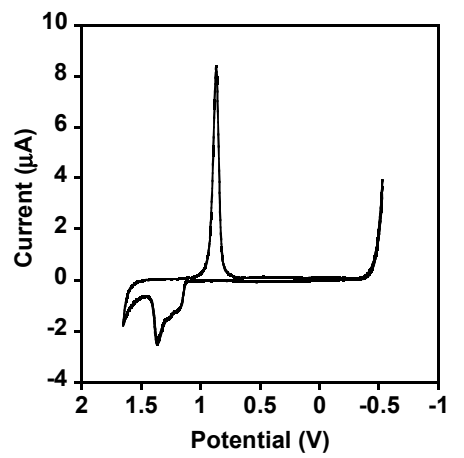

Figure 6. Electrochemical Cleaning of Gold Electrode.
Cyclic voltammetry was carried out in an electrochemical cell using a Bioanalytical Systems (BAS, Model CV-50W) electrochemical analyzer. A normal three-electrode configuration consisting of a modified gold-disk working electrode, a saturated calomel reference electrode (SCE, Fisher Scientific), and a platinum wire auxilliary electrode was used. The working compartment of the electrochemical cell was separated from the reference compartment. Potentials were reported versus SCE. Volumes of $30 \mathrm{ml}$ were typically employed. Figure 6 shows a typical cyclic voltammogram obtained on a gold microelectrode (1 $\mathrm{mm}$ in diameter) in $1 \mathrm{M} \mathrm{H}_{2} \mathrm{SO}_{4}$ upon cycling the potentials between $+1.65 \mathrm{~V}$ and $-0.5 \mathrm{~V}$ at a scan rate of $100 \mathrm{mV} / \mathrm{s}$. The sharp rise in anodic current to a peak near $+1.1 \mathrm{~V}$, a single oxide stripping peak near $+0.9 \mathrm{~V}$, and reproducible cyclic voltammograms on successive scans indicated that the gold surface was very clean and ready for DNA monolayer formation.

\section{DNA Film Formation}

15- to 21-base oligonucleotides immobilized on a controlled pore glass resin were first synthesized according to automated solid-phase techniques on an ABI 394 DNA/RNA Synthesizer and then treated in succession with carbonyldiimidazole and 1,6diaminohexane at the 5'-hydroxy terminus before cleavage from the resin. After deprotection, the free amine was treated with 2pyridyldithiopropionic acid $\mathrm{N}$-succinimide ester to produce a disulfide. Sequences were purified by reverse-phase HPLC, converted to free thiols using dithiothreitol, and repurified before hybridization to their complements. Gold microelectrodes were modified by incubation in $0.1 \mathrm{mM}$ solutions of derivatized DNA duplexes in $5 \mathrm{mM}$ phosphate/50 $\mathrm{mM} \mathrm{NaCl} / 0.1 \mathrm{M} \mathrm{Mg}^{2+}(\mathrm{pH}$ 7) for 12-24 hours at ambient temperature.

Comparison of cyclic voltammetry of ferricyanide at bare and DNA-modified gold microelectrodes provides a convenient and qualitative way to monitor the surface coverage of DNA duplexes. The negatively charged ion $\left[\mathrm{Fe}(\mathrm{CN})_{6}\right]^{4-}$ is repelled from the surface of the modified microelectrode by the polyanionic DNA, and should exhibit essentially no response when the surface is well covered. Figure 7 shows a typical cyclic voltammogram of a DNA modified gold microelectrode $(0.5 \mathrm{~mm}$ in diameter $)$ in $1 \mathrm{mM}$ $\left[\mathrm{Fe}(\mathrm{CN})_{6}\right]^{4-} / 2.5 \mathrm{mM}$ phosphate $/ 25 \mathrm{mM} \mathrm{NaCl}$. Indeed, the microelectrode well covered with DNA duplexes showed no electrochemical response whereas the bare electrode gave wellresolved redox peaks of ferricyanide.

\section{Mismatch Detection}

To demonstrate mutation detection, DNA-mediated electron transfer on gold microelectrodes was studied using cyclic voltammetry and chronocoulometry experiments. Single-base mismatches were incorporated into two different DNA duplexes

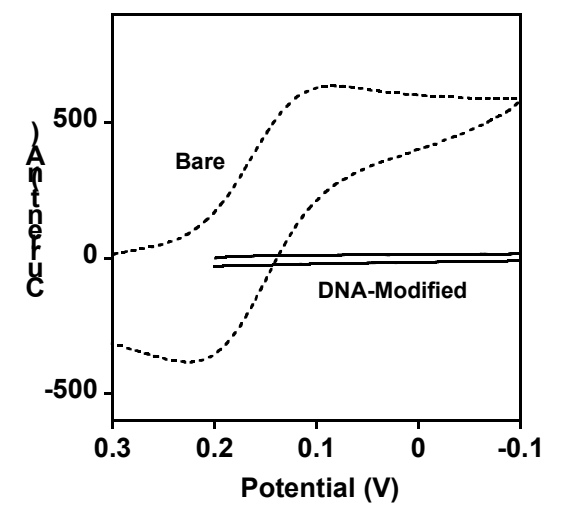

Figure 7. Blocking of Ferricyanide Redox by DNA Duplexes. 

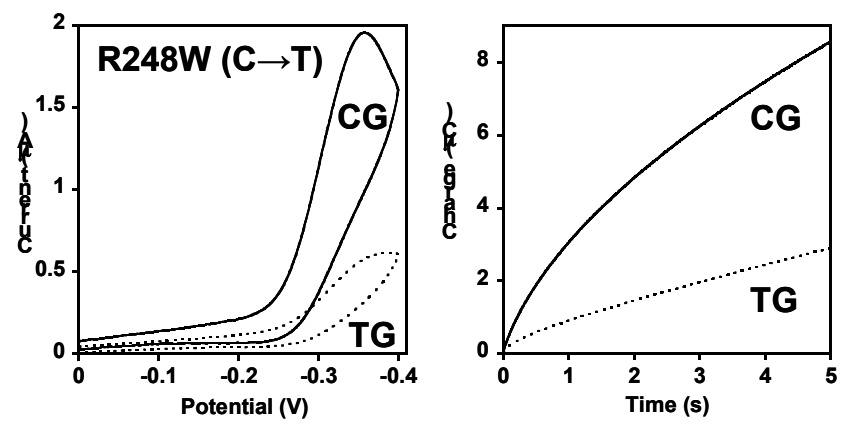

(a) 5'-ATGAACCGGAGGCCC-3' (C $\rightarrow \boldsymbol{T})$

Detection Condition: 0.25 $\mu M M B, 2$ mM Ferricyanide
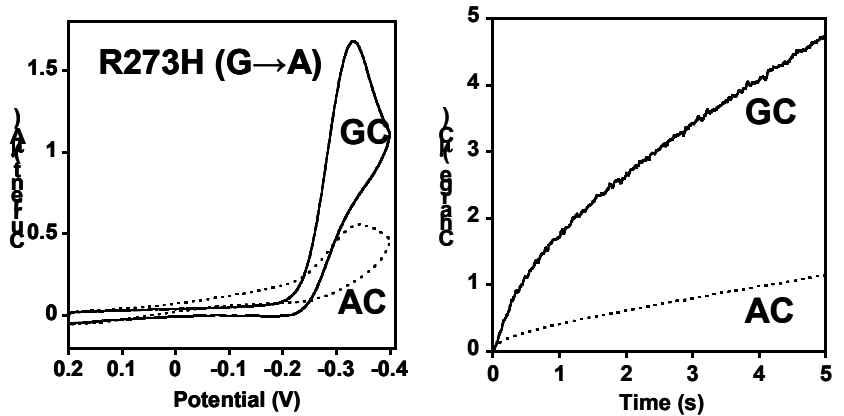

(b) 5'-GAGGTGCGTGTTTGT-3' (G $\rightarrow \boldsymbol{A})$

Detection Condition: $0.5 \mu M M B, 1 \mathrm{mM}$ Ferricyanide

Figure 8. Detection of Mismatches in p53 Gene.
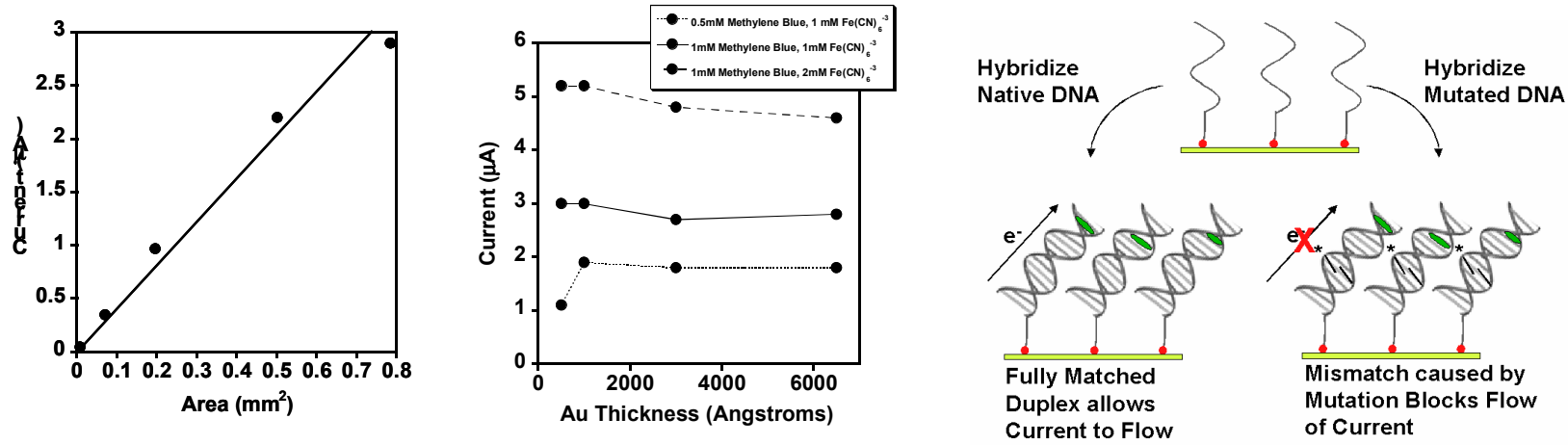

Figure 9. Dependence of Peak Current on Electrode Dimensions.

Figure 10. Electrochemical Microsensors for Mutation Detection.

(Figure 8). Gold microelectrodes modified with these duplexes were interrogated by cyclic voltammetry (potential between +0.2 to $-0.4 \mathrm{~V}$ and scan rate of $100 \mathrm{mV} / \mathrm{s}$ ) and chronocoulometry (potential at $-0.35 \mathrm{~V}$ ) with $\mathrm{MB}$ as the intercalated catalyst and ferricyanide as the solution substrate. The experimental results shown in Figure 8 clearly demonstrate the remarkable sensitivity of this electrocatalytic assay. Indeed, incorporation of a mismatch into the duplex significantly attenuated the electrocatalytic response. In both cases, the peak current and the measured charge at the fixed potential for base-paired duplexes were almost four times the ones for mismatched duplexes. It should be noted that because the charge transfer-based assay features a catalytic reaction whose rate depends on the degree of complementarity within the individual duplexes, the measured charge resulting from the reduction of $\mathrm{MB}$ at base-paired versus mismatched films increases disproportionately with longer integration time. Increased integration times continue to increase the differentiation of signals obtained with mismatched versus paired complements.

The effect of dimensions of gold microelectrodes on the electrocatalytic assay was also studied. Cyclic voltammetry of DNA-modified gold microelectrodes with different diameters $(0.1$ $\mathrm{mm}, 0.3 \mathrm{~mm}, 0.5 \mathrm{~mm}, 0.8 \mathrm{~mm}$, and $1 \mathrm{~mm}$ ) and different thicknesses $(500 \AA, 1000 \AA, 3000 \AA$, and $6500 \AA$ ) were performed. Figure 9 shows that the peak current is linearly proportional to the area, but shows no dependence on the thickness of the gold microelectrodes when the thickness is above $1000 \AA$.

\section{Electrochemical Microsensors}

Based on these results, we are developing microsensors for diagnosing mutations in individual patients (Figure 10). DNA duplexes are formed on the sensor electrode surface. The composition of the duplexes positions them to determine if DNA from a patient contains a mutation. One strand of the duplex - the strand linked to the electrode - is chemically synthesized with sequence pre-programmed to be a portion of a gene that is known to contain a mutational hot-spot. The second strand of the duplex is processed from a patient sample (e.g. blood sample or tumor biopsy). The duplexes form by hybridization (in 100\% yield) when the DNA-modified electrode is exposed to a solution of the patient's DNA duplexes. The readout of the mutation is performed using chronocoulometry with $\mathrm{MB}$ and ferricyanide. The amount of measured charge at a fixed potential determines the existence of a mismatch in the film which is caused by the mutation in the patient's sample.

\section{CONCLUSIONS}

We have demonstrated that DNA-mediated electron transfer reactions are exquisitely sensitive to the stacking of the intervening bases. Because of its insensitivity to mismatch position, mismatch identity, and sequence content, this electrochemical assay provides an accurate, rapid, and inexpensive solution to mutation analysis. Based on these results, we have developed microfabricated electrochemical sensors for detecting mutations in DNA duplexes.

\section{REFERENCES}

1. S.P.A. Fodor, "Massively Parallel Genomics," Sciences, 277, 393 (1997).

2. E.M. Southern, "DNA Chips - Analyzing Sequence by Hybridization to Oligonucleotides on a Large Scale," Trends in Genetics, 12, 110 (1996).

3. S.O. Kelley, R.E. Holmlin, E.D.A. Stemp, and J.K. Barton, "Photoinduced Electron Transfer in Ethidium-Modified DNA Duplexes: Dependence on Distance and Base Stacking," Journal of the American Chemical Society, 119, 9861 (1997).

4. S.O. Kelley, J.K. Barton, N.J. Jackson, L.D. McPherson, A.B. Potter, E.M. Spain, M.J. Allen, and M.G. Hill, "Orienting DNA Helices on Gold Using Applied Electric Field," Langmuir, 14, 6781 (1998). 